\title{
El impacto de las tecnologías de la información y comunicación en la industria manufacturera de Baja California
}

The impact of information and communication technologies on the manufacturing industry of Baja California

\author{
Lourdes Alicia González Torres* \\ Manuel Alejandro Ibarra Cisneros* \\ Karla Emilia Cervantes Collado*
}

Recibido el 19 de junio de 2015

Aceptado el 13 de enero de 2016

Resumen: en este artículo se analizó el comportamiento de la adopción de las tecnologías de la información y comunicación en los subsectores manufactureros de Baja California, para determinar sus efectos en los resultados económicos y los cambios que generan en las empresas. Para ello se utilizaron seis dimensiones del modelo desarrollado por Karmarkar y Mangal (2004), y con la tau-b de Kendall se calculó la correlación entre el grado de adopción de dichas tecnologías y los resultados económicos. Aunque éstos no se compararon con los de otros estudios, puesto que aquí sólo se seleccionó cierto tipo de empresas, se logró exponer la situación del sector manufacturero estatal, en relación con el uso de las tecnologías. Por lo que se puede concluir que

\footnotetext{
* Profesores-investigadores de la Facultad de Ciencias Administrativas de la Universidad Autónoma de Baja California. Río Nuevo y Eje Central s/n, zona del Río Nuevo, C. P. 21330 , Mexicali, Baja California, México.Teléfono (686) 582 3323. Correos electrónicos: aliceinsky@yahoo.com / manuel_ibarra@uabc.edu.mx / kkarla@uabc.edu.mx
} 
la adopción de éstas influye positivamente en los resultados de las empresas.

Palabras clave: correlación; industrias manufactureras; industria-innovaciones tecnológicas; sector industrial; pequeña y mediana empresa; investigación cualitativa.

Abstract: this paper analyzes the performance of the adoption of information and communication technologies in manufacturing sub-sectors of Baja California in order to determine their effects on the economic results and the changes generated in the companies. For this purpose, six dimensions of the model developed by Karmarkar and Mangal (2004) were utilized, and using the Kendall's tau-b the correlation between the level of adoption of such technologies and the economic results was calculated. Although these were not compared to those of other studies, since only certain types of companies were selected, the situation of state manufacturing sector was set out in relation to the use of these technologies. Therefore, it can be concluded that the adoption of such technologies has a positive influence on the performance of companies.

Key words: correlation; manufacturing industries; industry-technological innovations; industrial sector; small and mediumsized enterprises; qualitative research.

\section{Introducción}

Las tecnologías de la información y comunicación (TIC) se han posicionado como factor preponderante en la competitividad mundial al influir positivamente en la productividad y, por extensión, en el crecimiento económico; los estudios empíricos de Oliner y Sichel (2000); Jorgenson et al. (2008); Thompson y Garbacz (2007); Wieck y Vidal 
(2010); de la Organización para la Cooperación y el Desarrollo Económicos, OCDE $(2012,2006$ y 2003) y del Foro Económico Mundial, FEM $(2012,2010$ y 2009) han reconocido los efectos positivos que generan tanto a nivel micro como macro. Debido a la evidencia empírica de éstos en el crecimiento económico, resulta interesante analizar si en Baja California la adopción de las TIC también está influyendo de manera positiva en el crecimiento de las empresas al hacerlo en la productividad. El objetivo general planteado aquí fue analizar el comportamiento de la adopción de las TIC de las pequeñas y medianas empresas (PYMES) en los subsectores de la industria manufacturera de Baja California, a fin de determinar su repercusión en la economía. Se analizaron las PYMES por su importancia en la generación de empleo y de riqueza en el estado, pues 99.5 por ciento de las empresas son micro, pequeñas y medianas (MIPYME), y emplean a 68 por ciento del personal ocupado (Secretaría de Desarrollo Económico, SEDECO 2014). Los objetivos de este trabajo, en lo relativo al uso de las TIC por los subsectores de la manufactura de Baja California, fueron: a) analizar su comportamiento al adoptarlas; b) identificar a los que las usan más; c) detectar los cambios que provocan en su organización interna; d) examinar su situación en el entorno global, como consecuencia al emplearlas; e) identificar las más utilizadas por las empresas, para interactuar con clientes y proveedores y f) correlacionar el grado de adopción de ellas con los resultados económicos, medidos a través de los ingresos.

La hipótesis es que el grado de adopción de las TIC está relacionado con los resultados económicos de los subsectores de la industria manufacturera de Baja California, medidos a través de los ingresos, por lo que se esperaría una correlación positiva y alta entre dichas variables.

Es importante conocer si las TIC están mejorando el desempeño de las empresas que integran los subsectores y, como consecuencia, el de la economía en general. El ramo manufacturero cobró importancia en México a raíz de la apertura comercial, cuando se consideró como el motor de crecimiento de la economía. En los últimos años ha experimentado cambios estructurales, y en la actualidad varias de sus industrias sobresalen a escala mundial, como la automotriz; en 2012, México se convirtió en el cuarto mayor exportador de automóviles, así como el tercero en instrumentos y aparatos médicos, y ocupó el 
doceavo lugar en equipo aeroespacial; Baja California es líder en la producción de estos dos últimos.

\section{Marco teórico}

En los últimos años, las TIC se han convertido en un elemento importante que contribuye al crecimiento y desarrollo económico, a través de la eliminación de costos de transacción, la eficientización de procesos productivos y administrativos y la eliminación de barreras que permite la globalización de los conocimientos, entre otros efectos. Se ha demostrado que la expansión en las redes de información y comunicación beneficia al mundo, y que el incremento de la inversión en infraestructura de telecomunicaciones repercute directamente en el crecimiento económico de algunos países pobres (Thompson y Garbacz 2007). Por lo que las inversiones en este rubro se consideran un verdadero motor del crecimiento y del empleo (Wieck y Vidal 2010).

Los efectos de las TIC se han medido desde perspectivas distintas en países, regiones, industrias y empresas. Asimismo, se ha analizado su contribución en el aumento de la productividad y en el crecimiento económico a partir de: a) la demanda, a través del efecto de la inversión en ellas o de su utilización y b) la oferta, que se refiere a producirlas, lo cual ha generado muchos debates acerca de la forma de medir sus efectos (Stiroh 2002). El caso de Irlanda, en la década de 1990, es una evidencia de los efectos positivos de la producción de TIC, ya que su crecimiento superó con mucho al de todos los países de la Unión Europea. También se obtuvieron buenos resultados en el este asiático y en Finlandia, que vieron la producción de TIC como medio para la prosperidad y el crecimiento económico (Zhen y Pitt 2003). Los estudios de Oliner y Sichel (2000); Jorgenson y Stiroh (2008); Thompson y Garbacz (2007) y Wieck y Vidal (2010) demuestran que las empresas que producen TIC crecen más que otras, ya que hay un incremento en el tamaño y la productividad del sector, así como en los efectos conexos de las industrias que les suministran insumos. Así, estas tecnologías contribuyen a aumentar la productividad total de los factores en el conjunto de la economía, 
al ayudar a las empresas a innovar y a mejorar su eficiencia general (OECD 2008 y 2000).

Desde el punto de vista de la demanda, generalmente se analizan los efectos directos e indirectos que la inversión y la utilización de las TIC generan sobre la productividad y el crecimiento. Cuando se mide el impacto de las TIC de manera directa se incluye el capital en la función producción, y se estima la ecuación de la contabilidad del crecimiento, que es la forma más utilizada para medirlo. Mientras que cuando se mide el impacto indirecto se incluyen las variables de capital humano, comercio, capital social y emprenderurismo.

La mayoría de los estudios parten del modelo neoclásico del crecimiento de Solow (1956), el cual plantea que el libre funcionamiento del mercado generará un proceso de convergencia económica, que identifica a factores del lado de la oferta como las causas del crecimiento económico y la explicación de sus disparidades. Asimismo, las aportaciones recientes del denominado modelo de crecimiento endógeno han dado lugar a una nueva teoría del crecimiento.

Otro aspecto relevante es cómo la globalización de las TIC ha originado cambios en el mercado laboral, se observa un aumento en el empleo directo e indirecto de especialistas en estas tecnologías en sectores distintos a ellas (OCDE 2012). Asimismo, Barrenetxe y Cardona (2013) encontraron, en su estudio de la Unión Europea, que en los últimos años los sectores con mayor incremento de empleo son los de alta tecnología y los relacionados con las Tic, los cuales se caracterizan por la escolaridad elevada de sus trabajadores.

Las TIC producen cambios importantes en las estructuras de mercado y en la competencia que enfrentan las empresas, a través de cuatro vías, como menciona García (2002): a) la competencia creciente, derivada de la reducción de barreras y creación de modos nuevos de distribución; b) los modelos empresariales innovadores, con menores costos y mayores exigencias de calidad; c) las formas diferentes de comprar y vender, con mayor personalización de productos y servicios y d) las necesidades nuevas de formación, ya que los procesos requieren calificaciones distintas. Por lo tanto, en esta investigación se puso un interés especial en analizar los cambios provocados por la implementación de tecnologías de la información y comunicación en la industria manufacturera de Baja California, entre las PYMES. 
Por otra parte, los estudios para el caso de México concluyen que la inversión en TIC favorece a las exportaciones de la industria manufacturera, sobre todo a las de menor intensidad tecnológica (Valderrama y Neme 2011). Se observan brechas regionales importantes en la adopción de TIC, que siguen el patrón de concentración de la actividad económica del país. Las regiones norte y centro gastan más en computadoras versus el producto interno bruto (PIB), ya que superan el promedio nacional, y su crecimiento económico es superior, mientras que en las sur-este, pacífico y oeste-centro esta relación es inferior al promedio, y también se identifican como de menor crecimiento (Tello 2008).

\section{La industria manufacturera}

En México, la industria manufacturera trasforma una gran diversidad de materias primas en artículos de consumo; el Instituto Nacional de Estadística y Geografía (INEGI) la clasifica en diez divisiones o subsectores, de acuerdo con el tipo de productos que elabora: I) alimentaria, de las bebidas y tabaco; II) textiles, prendas de vestir y productos del cuero; III) madera; IV) papel, impresión y conexas; v) derivados del petróleo y del carbón, química del plástico y hule; vI) fabricación de productos a base de minerales no metálicos; VII) metálica; VIII) maquinaria y equipo; IX) fabricación de muebles y productos relacionados y $\mathrm{x}$ ) otras.

El sector manufacturero en México se ha convertido en el motor de crecimiento económico a raíz de la apertura económica del país, la cual generó mayor competencia que le permitió a la industria nacional desarrollar un proceso intenso de modernización de su planta productiva, y ha fomentado la especialización permitiendo el aprovechamiento óptimo de las ventajas comparativas en algunas áreas de la producción (Huerta y Chávez 2003).

Entre las industrias manufactureras de mayor crecimiento se encuentran la de instrumentos y aparatos médicos, que pertenece a la clasificación x y la automotriz y aeroespacial, que corresponde a la VIII. En 2012, el sector automotriz experimentó un gran crecimiento, que colocó a México en el cuarto mayor exportador de automóviles 
del mundo; siete de los diez fabricantes más grandes tienen plantas en el país. La industria aeroespacial ubica a México en el doceavo lugar en la exportación de equipo de este ramo y en el tercero de instrumentos y aparatos médicos en el mundo (Federal Reserve Bank of Atlanta 2013). Otro aspecto digno de destacar es que la industria automotriz se concentra en las regiones norte y centro, las de mayor crecimiento; la primera aporta 47 por ciento del PIB manufacturero total, y 43 la segunda (Banco Bilbao Vizcaya Argentaria 2012).

Datos del INEGI (2012) indican que en 2011 el sector industrial en México representó 36 por ciento del PIB nacional; del cual, 50 correspondió al manufacturero. El comportamiento de las divisiones en este sector no ha cambiado mucho en los últimos años; en 2011, la industria alimentaria, de las bebidas y tabaco contribuyó con 28 por ciento al PIB manufacturero nacional, y la de maquinaria y equipo, con 27; también la de derivados del petróleo y del carbón, la química del plástico y hule, así como la de industrias metálicas hicieron una aportación importante.

En 2011, el sector industrial de Baja California representó 34 por ciento del PIB estatal, del cual 66 por ciento correspondió al de las manufacturas, cuya aportación al PIB nacional fue de 3.3, y el subsector que más aportó fue el de maquinaria y equipo, con 46 por ciento, seguido de la industria alimentaria, de las bebidas y tabaco, con 19 (INEGI 2012). Estos datos demuestran la especialización industrial del estado, que destaca a escala nacional al poseer fuertes ventajas competitivas en los productos electrónicos principalmente. La contribución del resto de subsectores fue baja, inclusive marginal, como el caso de la industria de la madera.

En la actualidad, la competitividad del sector manufacturero resulta un elemento esencial para el crecimiento económico de un país, por sus efectos multiplicadores. En el periodo de 1980 a 2003, el Centro de Estudios de las Finanzas Públicas (2004) estimó que para México dichos efectos fueron moderados en los servicios, ya que por cada punto porcentual que creció el PIB manufacturero, los servicios lo hicieron en 0.8 por ciento en el largo plazo y en 0.4 en el corto, mientras que el del agropecuario fue de 0.3 y 0.2 por ciento respectivamente. Un sector manufacturero fuerte impulsa el capital intelectual y la capacidad de innovación de un país (Deloitte 2010). Por lo 
tanto, resulta importante analizar los factores que pueden contribuir a que sea más competitivo y que genere un efecto positivo en el crecimiento económico.

\section{Metodología}

Esta investigación es producto del proyecto "La adopción de las TIC en las pequeñas y medianas empresas del sector manufacturero de Baja California”, financiado por el Programa de Mejoramiento del Profesorado, de la Secretaría de Educación Pública, cuya metodología se basa en Ibarra et al. (2013). Aquí se buscó profundizar en el conocimiento existente sobre el uso de las TIC en la economía nacional y en la sectorial. En este artículo se revisaron los subsectores manufactureros para analizar la repercusión de las TIC en los resultados económicos y, a su vez, en las empresas de esta industria tan relevante para Baja California. Para lograr este objetivo se utilizaron las seis dimensiones del modelo desarrollado por Karmarkar y Mangal (2004), quienes desde 2002 han analizado cómo se encuentra la adopción de las TIC en países como Estados Unidos, India, Italia, España y Corea del Sur, entre otros, en los rubros del comercio al por menor, el hipotecario, las telecomunicaciones, el financiero y el automotriz. Para esta investigación se seleccionó a las PYMES, y se adaptó el modelo a sus necesidades. Por tanto, los resultados presentados aquí no se compararon con estudios de los autores citados, ya que sólo se eligió a cierto tipo de empresas estatales. La primera dimensión fue la adopción de tecnología o infraestructura actual y su tendencia a futuro; se centró en conocer los tipos de TIC adoptados por las empresas, y en analizar la tendencia a incorporar nuevos. La segunda correspondió a la organización interna; referida a los cambios en la estructura, derivados de la implementación de las TIC, es decir, si existía una tendencia a crear áreas y departamentos, al igual que los cambios y comportamientos en la fuerza laboral, los mecanismos de comunicación interna y la resistencia al cambio. La tercera fue el impacto de la interacción con los clientes; se enfocó en analizar los medios de TIC con los que cuenta la empresa para interactuar con sus clientes, así como los más utilizados. También se investigaron las TIC más apropiadas para vender o 
promocionar sus productos. En la cuarta se revisó el impacto de la interacción con los proveedores; consistió en analizar los medios de TIC de la empresa para interactuar con ellos, así como los más utilizados y más eficientes. La quinta fue el impacto en los resultados del negocio; buscó determinar en qué medida la adopción de las tecnologías ayuda a las empresas a reducir costos, al mejoramiento financiero y a disminuir aspectos operativos. Por último, la dimensión referente a la globalización permitió conocer si la adopción de las TIC ha sido positiva para el conocimiento estratégico de las empresas, si conocían más el mercado internacional, a los clientes, a los competidores y las oportunidades de negocio en otros países.

El diseño de la investigación fue no experimental, de tipo cuantitativo, descriptivo-correlacional y de forma trasversal. Se diseñó un cuestionario de 67 reactivos distribuidos en seis dimensiones. Para la deter-

\section{Figura 1}

Distribución de encuestas aplicadas

\begin{tabular}{|c|c|c|c|}
\hline $\begin{array}{c}\text { Subsectores de la industria } \\
\text { manufacturera de Baja California }\end{array}$ & Subsector & $\begin{array}{c}\text { Total de } \\
\text { empresas }\end{array}$ & $\begin{array}{c}\text { Porcentaje de empresas } \\
\text { encuestadas }\end{array}$ \\
\hline $\begin{array}{c}\text { Alimentaria, } \\
\text { de las bebidas y tabaco }\end{array}$ & I & 219 & 22.3 \\
\hline $\begin{array}{c}\text { Textiles, prendas de vestir } \\
\text { y productos del cuero }\end{array}$ & II & 205 & 7.4 \\
\hline $\begin{array}{c}\text { Madera, fabricación de muebles } \\
\text { y productos relacionados }\end{array}$ & III & 117 & 4.7 \\
\hline $\begin{array}{c}\text { Papel, impresión y conexas } \\
\text { química del plástico y hule }\end{array}$ & IV & 109 & 10.8 \\
\hline $\begin{array}{c}\text { Fabricación de productos } \\
\text { a base de minerales no metálicos }\end{array}$ & VI & 48 & 10.8 \\
\hline Metálicas & VII & 29 & 7.4 \\
\hline Maquinaria y equipo & VIII & 381 & 16.2 \\
\hline Otras & IX & 171 & 17.7 \\
\hline
\end{tabular}

* Estos subsectores se fusionaron debido a que sólo se encuestó a una empresa y su análisis por separado no era representativo a escala estatal.

Fuente: elaboración propia. 
minación de la muestra estadística se usó el padrón de empresas del sector manufacturero de la Secretaría de Desarrollo Económico del Estado de Baja California (2014), y el Directorio estadístico nacional de unidades económicas del INEGI (2016). Después se depuraron ambas bases, y se creó la propia, esto debido a que las consultadas no estaban actualizadas. La población de las PYMES se determinó con la clasificación establecida por la Secretaría de Economía y la Secretaría de Hacienda y Crédito Público en México, referente al número de empleados. Para las empresas pequeñas del sector industrial se consideró a las de entre 11 y 50 empleados, mientras que para una mediana, de 51 a 250. Las PYMES detectadas en el estado fueron 1 438, en la figura 1 se muestra el total de ellas por subsector; a partir de esto se determinó una muestra de 264, también aparece el porcentaje de las encuestadas (con 5 por ciento de margen de error y 95 de confianza, y con un valor p de .70 -obtenido de la prueba piloto-), de la cual se encuestó a 191, 72 por ciento de la esperada, debido a que muchas no participaron o no se localizaron.

El análisis descriptivo de los resultados se realizó a través de tablas de contingencia. Asimismo, se calculó el coeficiente de correlación tau-b de Kendall del grado de adopción de TIC y el incremento de los ingresos de las empresas. Para probar la hipótesis de que el grado de adopción de tecnologías de la información y comunicación está relacionado con los resultados económicos de los subsectores de la industria manufacturera de Baja California, medidos a través de los ingresos, se utilizó la prueba de hipótesis tau-b de Kendall, que es la adecuada cuando las variables que se intenta correlacionar son dos ordinales.

Existen tres medidas de Kendall, la tau-a, la tau-b y la tau-c; la primera está basada en que todos los pares posibles están clasificados (no relacionados); este caso es raro en la práctica, por lo tanto, lo común es calcular la tau-b. La tau-c es una versión ajustada de la tau-b, para tablas con número de renglones y columnas diferentes (Menard 2009).

Aquí se utilizó la tau-b debido a que el número de columnas y renglones era igual.

La tau-b de Kendall es una medida no paramétrica de asociación para variables ordinales o de rango, cuya asociación se basa en el número de concordancias y discordancias de pares de observaciones; 
cuantifica el grado de concordancia monótona entre dos variables ordinales (Laurencelle 2009, 52).

Dos observaciones $\left(\mathrm{X}_{\mathrm{i}}, \mathrm{Y}_{\mathrm{i}}\right)$ y $\left(\mathrm{X}_{\mathrm{j}}, \mathrm{Y}_{\mathrm{j}}\right)$ son concordantes si tienen el mismo orden con respecto a cada una de las variables. Esto es si:

(1) $X_{i}<X_{j} y Y_{i}<Y_{j}$, o si

(2) $X_{i}>X_{j} y Y_{i}>Y_{j}$

Estas son discordantes si el orden es inverso para X y $\mathrm{Y}$, o los valores están dispuestos en direcciones opuestas. Esto es si:

(1) $X_{i}<X_{j} y Y_{i}>Y_{j}$, o si

(2) $X_{i}>X_{j} y_{i}<Y_{j}$

Dos observaciones están relacionadas si $X_{i}=X_{j} \circ Y_{i}=Y_{j}$.

El número total de pares que se puede construir para una medida de muestra $\mathrm{N}$ es:

$$
\mathrm{N}=\left(\frac{n}{2}\right)=\frac{1}{2} \mathrm{n}(\mathrm{n}-1)
$$

$\mathrm{N}$ se puede descomponer en cinco cantidades

$$
\mathrm{N}=\mathrm{P}+\mathrm{Q}+\mathrm{X}_{0}=\mathrm{Y}_{0}+(\mathrm{XY})_{0}
$$

donde $\mathrm{P}$ es el número de pares concordantes, $\mathrm{Q}$ el de pares discordantes, $\mathrm{X}_{0}$ es el de los relacionados sólo en la variable $\mathrm{X}, \mathrm{Y}_{0}$ es el de los relacionados en la variable $\mathrm{Y}$, y $(\mathrm{XY})_{0}$ es el de los relacionados en ambas, X y Y.

La tau-b de Kendall para las medidas de asociación entre variables X y Y está dado por la fórmula:

$$
\mathrm{T}_{\mathrm{b}}=\frac{\mathrm{P}-\mathrm{Q}}{\sqrt{(\mathrm{P}+\mathrm{Q}+\mathrm{X} 0)(\mathrm{P}+\mathrm{Q}+\mathrm{Y} 0)}}
$$

El valor se encuentra entre -1 y +1 (Penn State Eberly College of Science 2015). El signo del coeficiente indica la dirección de la relación y su valor absoluto la magnitud de ella, de tal modo que los mayores valores absolutos indican correlaciones más fuertes. El coeficiente de correlación tau-b de Kendall puede tomar valores de -1 a +1 , pero el de -1 o +1 sólo se puede obtener en tablas cuadradas, es decir, con el mismo número de filas y columnas. 
El coeficiente tau-b de Kendall es similar al coeficiente de correlación producto momento de Spearman y Pearson. Los valores negativos indican que cuando $\mathrm{X}$ se incrementa $\mathrm{Y}$ decrece, es decir, los resultados de ambas variables van en dirección contraria, mientras que los valores positivos indican que los datos en las dos son congruentes en la misma dirección y, como ocurre en la correlación de Pearson y Spearman, cuando más cercano a 1 o -1 sea el coeficiente tau-b, más fuerte será la asociación, mientras que, cuanto más cercana a 0 , la asociación es más débil. El coeficiente tau-b de Kendall representa una probabilidad, es la diferencia entre que los datos observados sean del mismo orden, contra la de que no lo sean (Statistical Research 2012).

\section{Análisis de resultados}

Primero se analizaron las dimensiones planteadas en el instrumento, después los resultados correspondientes al coeficiente y la prueba de hipótesis tau-b de Kendall sobre la existencia de correlación entre el grado de adopción de TIC y los resultados económicos de los subsectores, medidos por los ingresos.

Análisis de los resultados de las dimensiones del modelo Adopción de tecnología o de la infraestructura actual y su tendencia a futuro

Los resultados muestran que el software más utilizado fue el relacionado con herramientas de administración del negocio (sistema tres, COMPAQ), seguido por el de control de virus y spam, y al final los administradores de bases de datos. Destacaron las divisiones 5 y 8 que usan, en mayor proporción, el software para el control de virus y spam. En lo que se refiere a las tendencias futuras, los subsectores no tenían contemplado invertir en adquisición de software nuevo, con excepción de las 5 y 7, que consideraban hacerlo en el largo plazo. En cuanto al hardware, los más usados fueron: computadoras, impresoras, copiadoras, proyectores y dispositivos de almacenamiento, en menor proporción contaban con servidores web, de correo y firewall, sobre- 


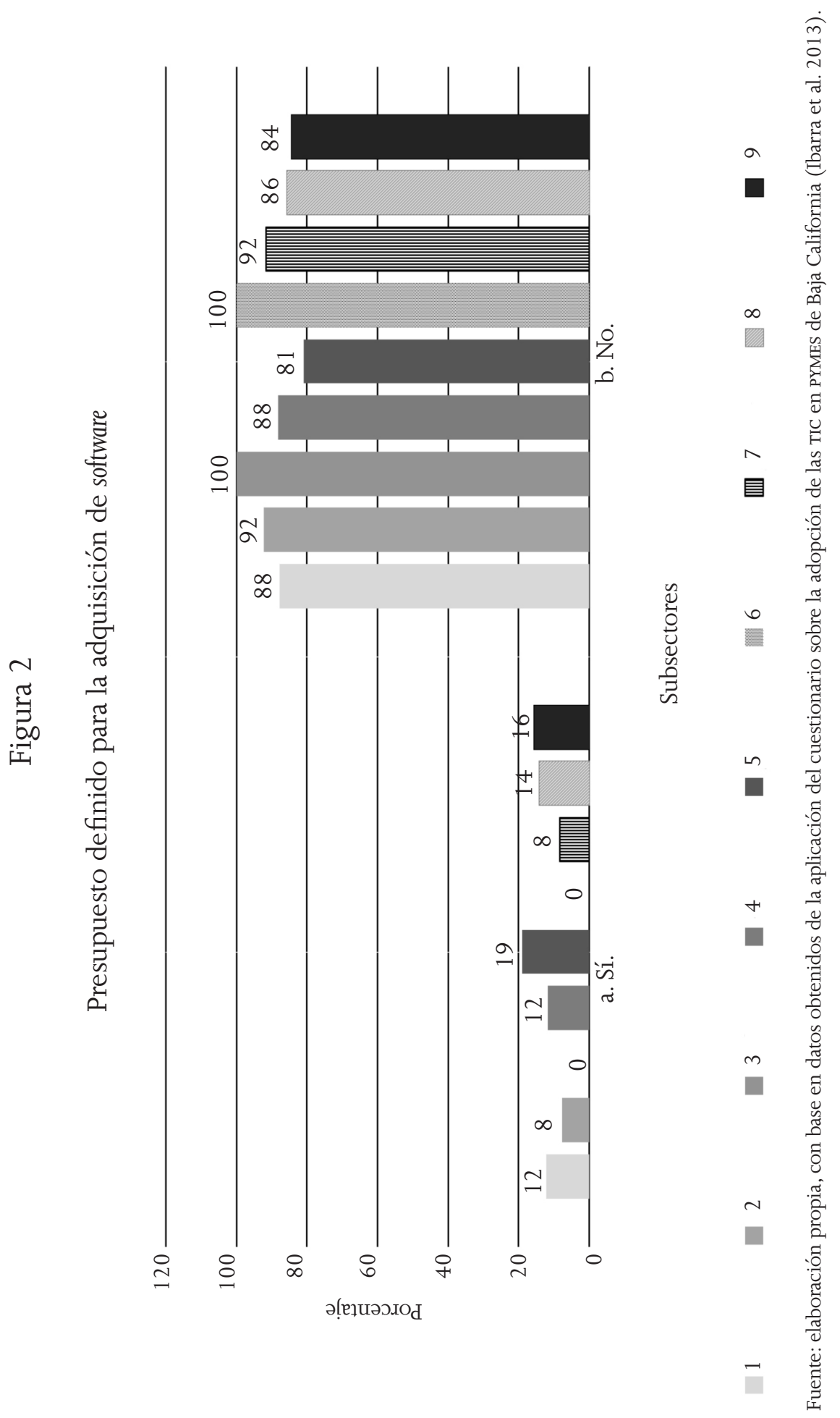


salieron los subsectores 4, 5, 8 y 9 por usar la mayor proporción y diversidad de hardware. En cuanto a si tenían contemplada la adquisición de hardware, todos los sectores planeaban comprar equipos, salvo el 1, 2 y 9; el 3 y 5 lo harían en menos de un año, mientras que el 4, 7 y 8 entre uno y tres. Los subsectores no contaban con presupuesto definido para la adopción de TIC, excepto unas cuantas empresas (véase figura 2).

\section{Organización interna}

En todos los subsectores de la dimensión in hubo cambios en algunos aspectos de la organización, relacionados con la adopción de las TIC, excepto en el 1,2 y 3 . Otro aspecto relevante consistió en saber si la implementación de las TIC contribuyó en esta dimensión a la creación o desaparición de puestos de trabajo; se encontró que no hubo creación ni desaparición de éstos. Tampoco se observaron cambios en el mercado laboral a raíz de la implementación de las TIC, pues no se incrementó la calificación de la mano de obra, lo que contrasta con lo descubierto en el estudio empírico de Krueger (1993), quien encontró que los trabajadores que utilizaban computadoras ganaban entre 10 y 15 por ciento más que los que no lo hacían, lo cual está asociado con personal que tiene más escolaridad. Sólo en los subsectores 7 y 8 fue amplia la proporción de empresas que consideraron que la calificación de la mano de obra había aumentado, como se muestra en la figura 3. Por lo tanto, se puede decir que los cambios moderados en la estructura organizacional en las empresas se refieren a efectos en la comunicación interna, más que a los cambios en el empleo y a la calificación de los trabajadores.

Impacto de la interacción con los clientes

En la dimensión III, el correo electrónico fue el medio más utilizado en todos los subsectores, excepto en el I, que usó más los mensajes por teléfono, el segundo más empleado, y después la página de internet (véase figura 4).

Los subsectores de la manufactura consideraron suficientes los medios tecnológicos empleados por las empresas, a pesar de que fueron muy pocos. Todos realizaban ventas en línea, pero en menos de 


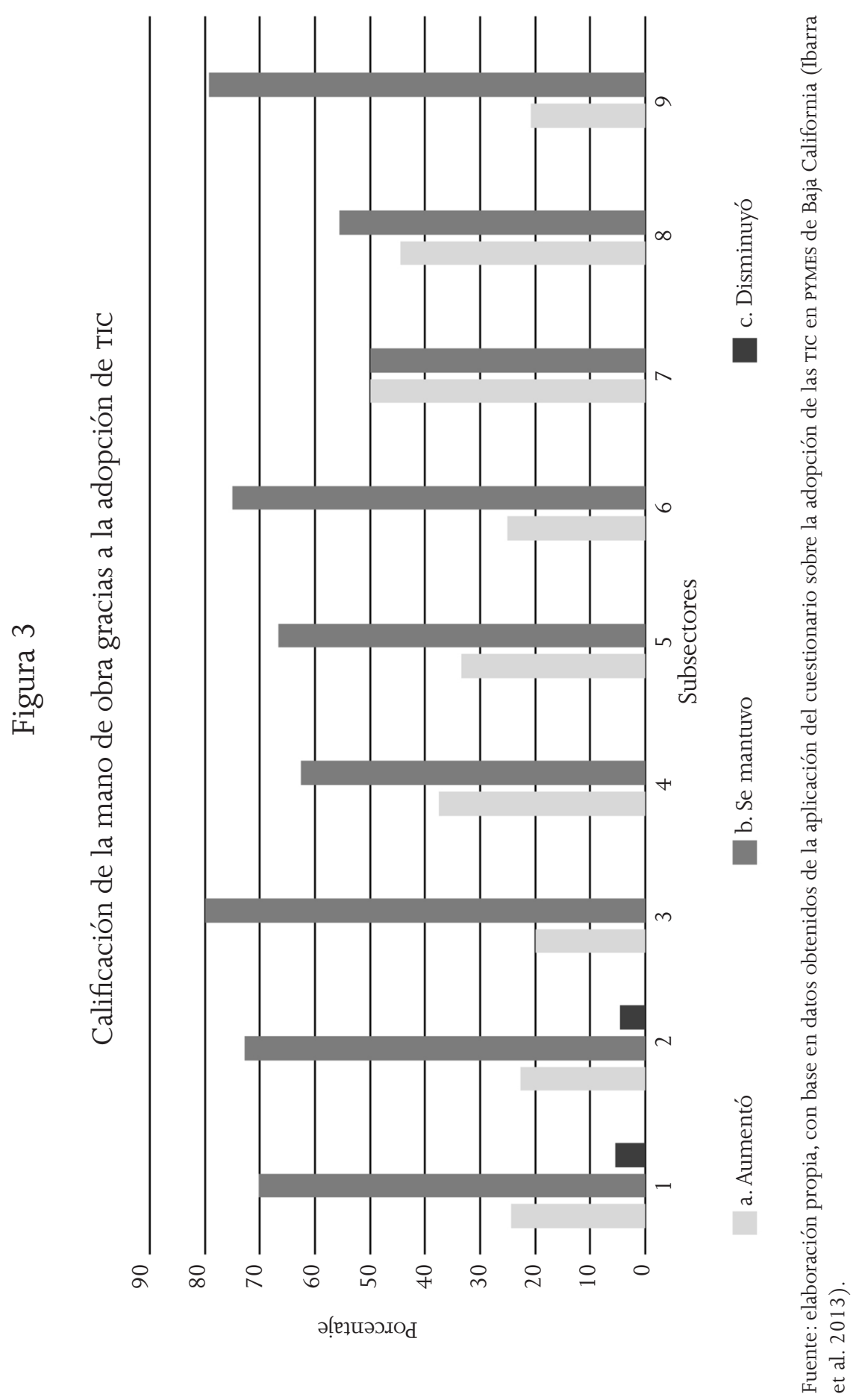




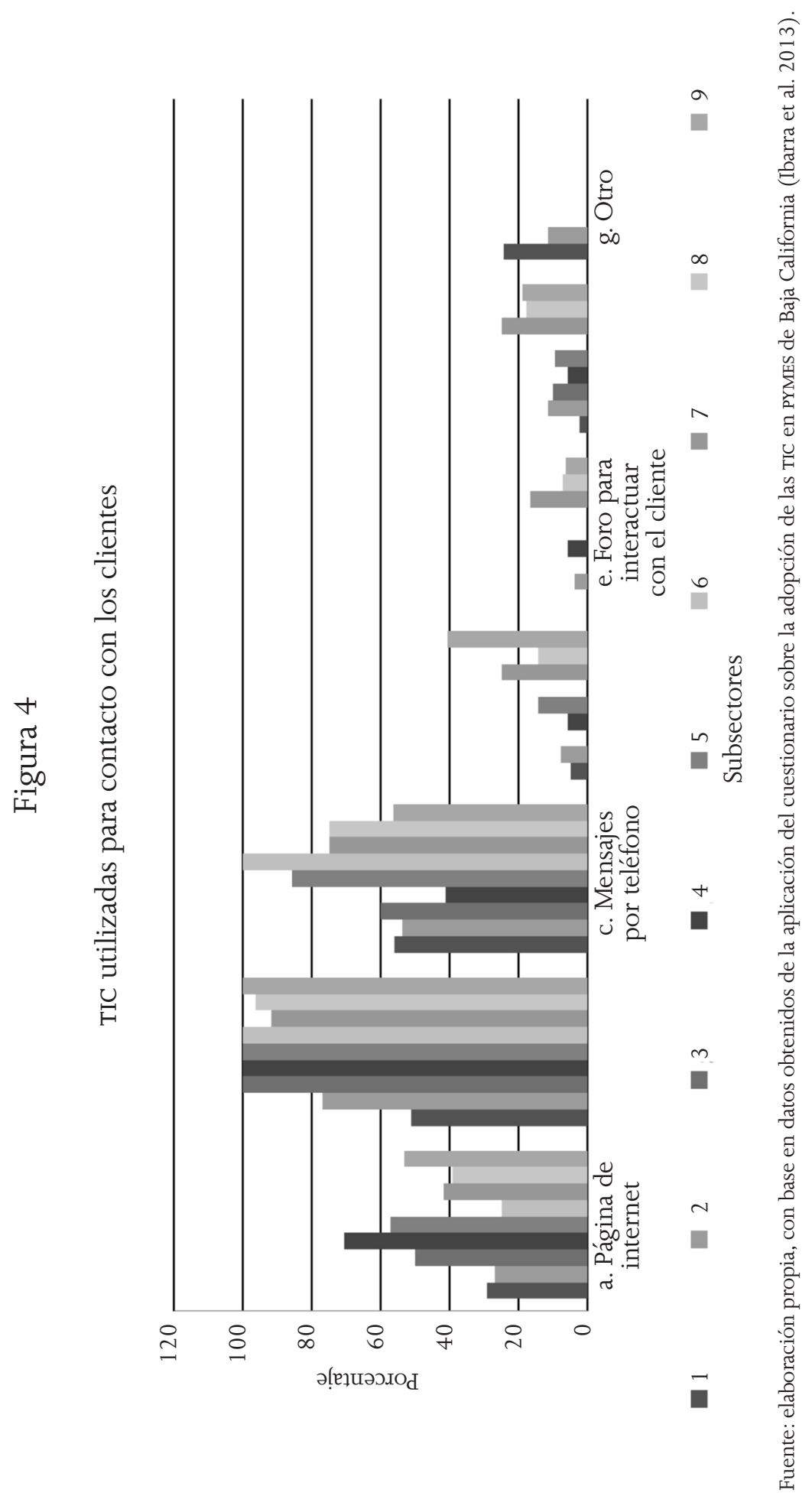




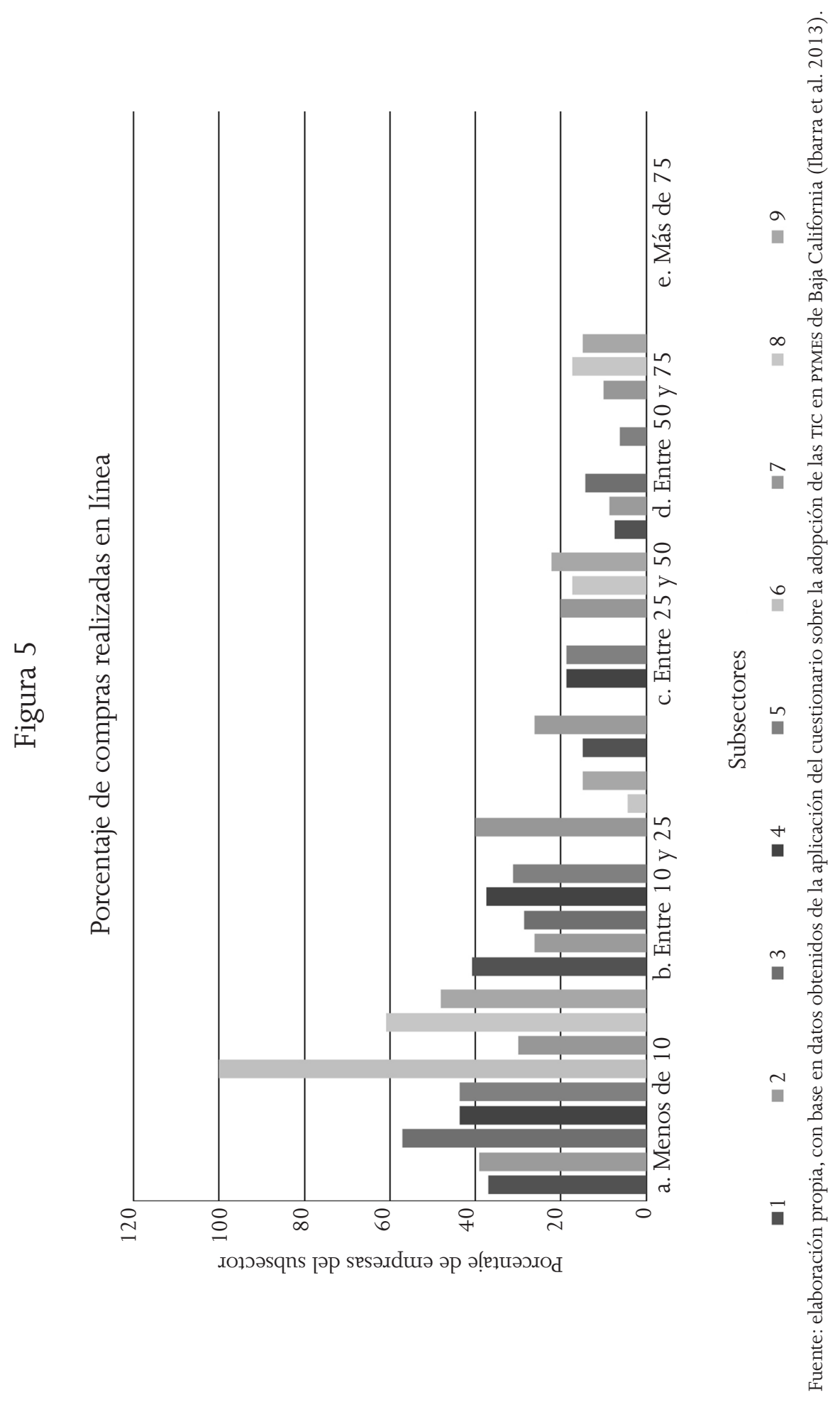


10 por ciento de las ventas totales. El cliente tiene una percepción positiva acerca de que la empresa use las TIC para hacer negocios, aunque los subsectores 1 y 3 se mostraron indiferentes ante esta percepción del cliente.

Impacto de la interacción con los proveedores

Para la dimensión IV, el correo electrónico también fue el medio de comunicación más utilizado para interactuar con los proveedores, seguido de los mensajes por teléfono y la página de internet. Asimismo, todas las empresas consideraron que los medios para comunicarse con sus proveedores fueron suficientes.

Un aspecto importante de esta dimensión es que las empresas compraban insumos y materiales en línea, aunque predominaron las que lo hacían algunas veces, luego las que las realizaban rara vez, y sólo el subsector 7 y 8 lo hacía casi siempre.

En cuanto al porcentaje de compras en línea, sólo los subsectores 1,7 y 9 compraban entre 10 y 15 por ciento por esta vía, mientras que todos los demás lo hacían en menos de 10 por ciento. Menos de 20 por ciento de las empresas compraba entre 25 y 50 por ciento en línea; destacaron los subsectores 2,7 y 9 que efectuaban 26, 20 y 22 por ciento de sus compras por este medio, mientras que un porcentaje mínimo realizaba entre 50 y 75 por ciento de sus compras de este modo, de nuevo sobresalieron los subsectores 3, 7, 8 y 9 que lo hacían en $14,10,17$ y 15 por ciento respectivamente (véase figura 5).

La mayoría de las empresas tuvieron una actitud positiva por parte de sus proveedores acerca de la utilización de las TIC en las negociaciones, sólo los subsectores 1, 2, 6 y 8 se mostraron indiferentes al respecto.

Impacto en los resultados del negocio

La mayoría de las empresas en la dimensión v consideró que sus ingresos habían aumentado de manera moderada, sólo en los subsectores 1,3 y 6 el incremento fue poco y mucho en el 7.

En lo que se refiere a la reducción de costos, derivados de la implementación de las TIC, ésta fue poca en los subsectores $1,2,3$, y 6, mientras que en los 4, 5, 7 y 9 fue moderada, y en 29 por ciento 


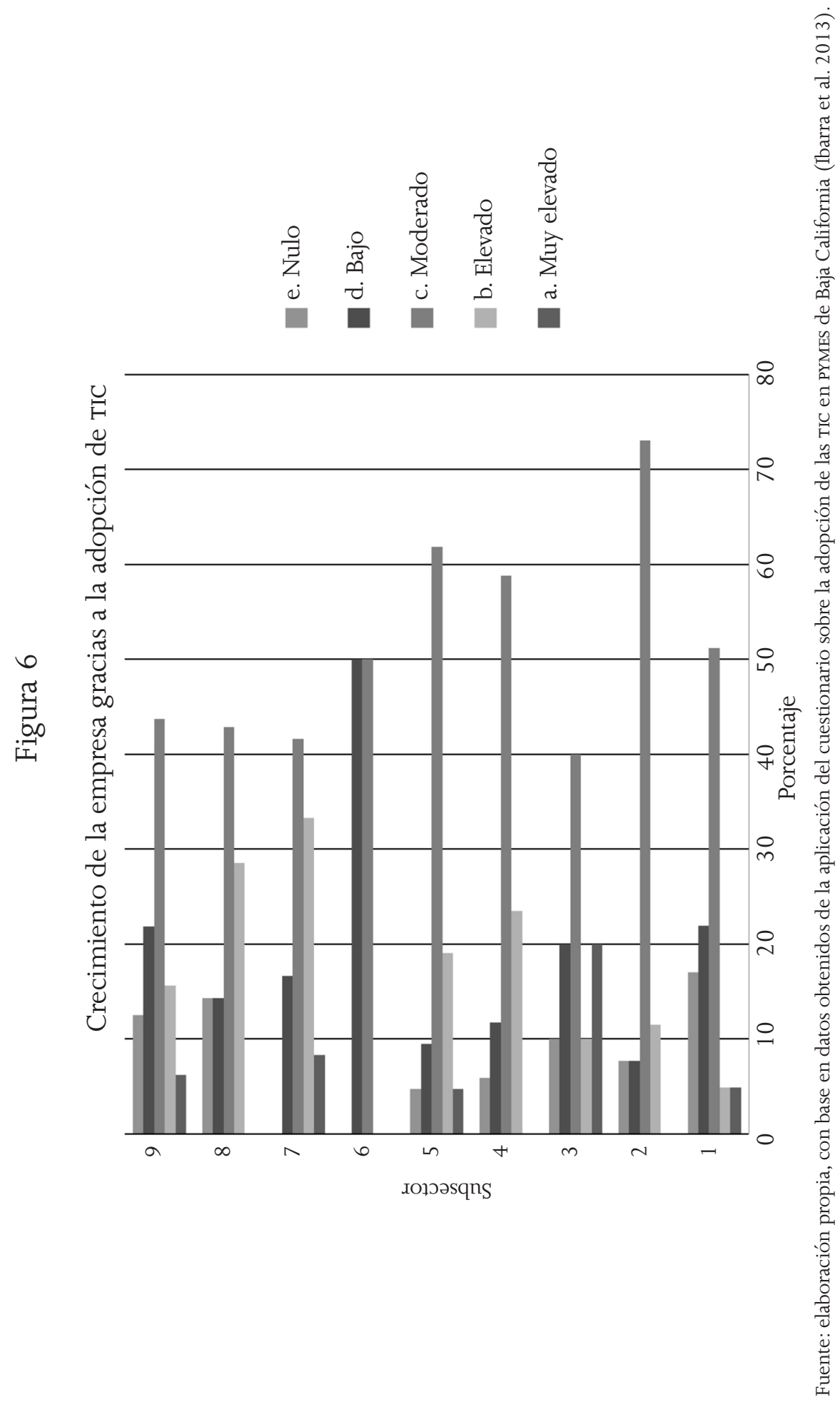


de las empresas del 8 se redujeron los costos en gran medida y un porcentaje igual consideró que la disminución fue poca. En cuanto al crecimiento de las empresas, gran parte de ellas consideró que fue moderado, gracias a la adopción de las TIC. Destacó un alto porcentaje de las de los subsectores 8,9 y 4 que consideró que su crecimiento había sido elevado, en tanto que para un gran porcentaje de las del 6 éste había sido bajo (véase figura 6).

En cuanto al diseño de estrategias de marketing, se encontró que en los subsectores 1, 2, 3, 4, 5 y 9 algunas veces las diseñaban en función de las Tic; el 7 siempre lo hacía, pero el 6 y el 8 nunca lo hicieron. Los departamentos que realizaban un mayor porcentaje de sus compras en línea fueron los de contabilidad, gerencia y ventas. En cuanto a cómo se percibían con respecto a la competencia dentro de su sector, en lo relativo a la adopción de las TIC, todas las empresas de los subsectores señalaron encontrarse al mismo nivel.

\section{Globalización}

Por último, todos los subsectores de la dimensión vi tenían comercio internacional; sin embargo, el mayor porcentaje de estas ventas lo realizaban los 2, 5, 6 y 9; el 8 tenía la mayor participación nacional, y el resto la regional.

El porcentaje de ventas de clientes extranjeros varió entre los subsectores, la mayoría vendía menos de 10 por ciento al exterior; y el 2 y el 9 más de 75. También destacó el 7, compuesto por empresas con características contrastantes, pues mientras 33 por ciento de ellas no vendía al exterior, 17 lo hacía en menos de 10 por ciento, y 17, entre 10 y 25 , y 17 por ciento, entre 50 y 75. El principal cliente externo de todos los subsectores es Estados Unidos.

En la parte gris de la figura 7 destacan los subsectores 4,8 y 9, que se han beneficiado, de manera importante, con la globalización de las TIC; mientras los 2, 5 y 7 lo han hecho ligeramente, y para el 1, 3 y 6 no ha tenido beneficio alguno.

Los principales competidores de todos los subsectores son nacionales, con excepción del 5, que consideró que procedían de Estados Unidos. Sin embargo, dicho país es el segundo competidor de todos los subsectores, y sólo para el 6 son China y Estados Unidos, con el mismo porcentaje que el mercado nacional. 


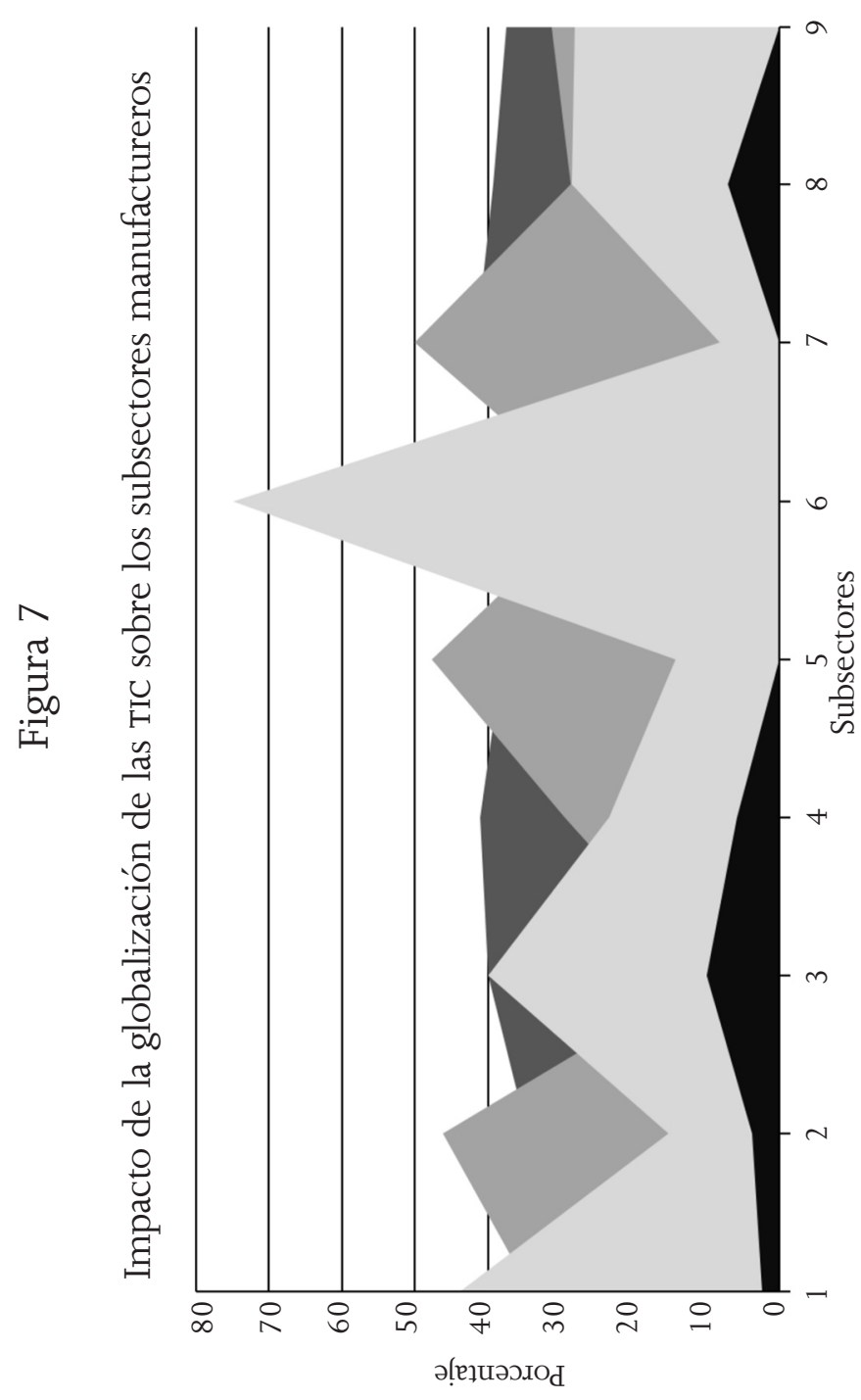

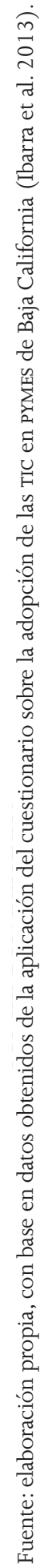




\section{Figura 8}

\section{Resultados principales}

\begin{tabular}{|c|c|}
\hline Dimensión & Resultados principales \\
\hline \multirow[t]{4}{*}{$\begin{array}{l}\text { Adopción de } \\
\text { TIC }\end{array}$} & $\begin{array}{c}\text { Escasa utilización de programas informáticos. Se usó principalmente } \\
\text { software de herramientas de administración del negocio; destacaron } \\
\text { los subsectores } 5 \text { y } 8 \text {, ya que también usaron una gran proporción } \\
\text { de software para el control de virus y spam }\end{array}$ \\
\hline & $\begin{array}{c}\text { Falta de prioridad en la adquisición de software, sólo los subsectores } \\
5 \text { y } 7 \text { contemplaron comprarlo en el largo plazo }\end{array}$ \\
\hline & Sólo se utilizaban equipos de cómputo y sus periféricos \\
\hline & Planeación tecnológica inexistente \\
\hline \multirow{3}{*}{$\begin{array}{l}\text { Organización } \\
\text { interna }\end{array}$} & $\begin{array}{l}\text { Estructuras organizacionales con pocos cambios derivados del uso } \\
\text { de las TIC: hubo una mejora en la comunicación interna }\end{array}$ \\
\hline & $\begin{array}{l}\text { No hubo creación ni desaparición de puestos, derivados } \\
\text { de la implementación de las TIC }\end{array}$ \\
\hline & $\begin{array}{l}\text { Sólo los subsectores } 7 \text { y } 8 \text { consideraron un aumento en la } \\
\text { calificación de la mano de obra debido al uso de las TIC }\end{array}$ \\
\hline \multirow{4}{*}{$\begin{array}{l}\text { Impacto en } \\
\text { interacción } \\
\text { con los } \\
\text { clientes }\end{array}$} & $\begin{array}{l}\text { El correo electrónico, seguido del teléfono y la página de internet } \\
\text { fueron los medios más utilizados para interactuar con los clientes }\end{array}$ \\
\hline & Las empresas consideraron suficiente el uso superficial de las TIC \\
\hline & Menos de 10 por ciento de la ventas se realizó en línea \\
\hline & $\begin{array}{c}\text { Todos los subsectores señalaron una percepción positiva por parte } \\
\text { del cliente acerca del empleo de las TIC en la empresa, para realizar } \\
\text { negocios. Sólo el } 1 \text { y el } 3 \text { mostraron indiferencia }\end{array}$ \\
\hline \multirow{4}{*}{$\begin{array}{l}\text { Impacto en } \\
\text { interacción } \\
\text { con los } \\
\text { proveedores }\end{array}$} & $\begin{array}{l}\text { El correo electrónico, seguido del teléfono y la página de internet } \\
\text { fueron los medios más utilizados para interactuar } \\
\text { con los proveedores }\end{array}$ \\
\hline & Satisfacción con el uso mínimo de las TIC \\
\hline & $\begin{array}{l}\text { Todos los subsectores compraban en línea, destacaron } \\
\text { el } 7 \text { y el 8, que lo hacían casi siempre }\end{array}$ \\
\hline & $\begin{array}{l}\text { La mayoría de las empresas realizaba menos de } 10 \text { por ciento } \\
\text { de sus compras en línea }\end{array}$ \\
\hline \multirow{6}{*}{$\begin{array}{l}\text { Impacto en } \\
\text { resultados } \\
\text { del negocio }\end{array}$} & $\begin{array}{l}\text { La repercusión de las TIC en los ingresos fue de baja a moderada, } \\
\text { sólo el subsector } 7 \text { consideró que fue grande }\end{array}$ \\
\hline & $\begin{array}{c}\text { Para los subsectores } 1,2,3 \text { y } 6 \text { hubo poca reducción de costos, } \\
\text { para el } 4,5,7 \text { y } 9 \text { fue moderada y el } 8 \text { tuvo una gran reducción, } \\
\text { a raíz del uso de las TIC }\end{array}$ \\
\hline & $\begin{array}{l}\text { El crecimiento de las empresas fue moderado; } \\
\text { los subsectores } 8,9 \text { y } 4 \text { tuvieron uno elevado }\end{array}$ \\
\hline & Bajo uso de mercadotecnia a través de las TIC \\
\hline & En ventas, contabilidad y compras se usaron más las TIC \\
\hline & Consideración de la competencia en el uso de las TIC al mismo nivel \\
\hline
\end{tabular}




\begin{tabular}{|c|c|}
\hline \multirow{4}{*}{ Globalización } & $\begin{array}{c}\text { Todos los subsectores tuvieron comercio internacional. Destacaron } \\
\text { el 2, 5, } 6 \text { y 9, cuya mayor proporción de ventas fue internacional, } \\
\text { el } 7 \text { tuvo más ventas nacionales y el resto regionales }\end{array}$ \\
\cline { 2 - 3 } & $\begin{array}{c}\text { Estados Unidos fue el cliente externo principal } \\
\text { fueron nacionales, con excepción del } 5 \text { que identificó } \\
\text { a Estados Unidos como su mayor competidor }\end{array}$ \\
\cline { 2 - 3 } & $\begin{array}{c}\text { El segundo mayor competidor de todos los subsectores } \\
\text { fue Estados Unidos }\end{array}$ \\
\cline { 2 - 3 } & $\begin{array}{c}\text { Para los subsectores } 4,7 \text { y } 9 \text { el beneficio de la globalización } \\
\text { de las tic fue importante, para el } 2,5 \text { y } 7 \text { fue ligero } \\
\text { y el } 1,3 \text { y } 6 \text { no lo percibió }\end{array}$ \\
\hline
\end{tabular}

Fuente: elaboración propia, con base en datos obtenidos de la aplicación del cuestionario sobre la adopción de las TIC en PYMES de Baja California (Ibarra et al. 2013).

Análisis de resultados de la aplicación de la prueba tau-b de Kendall

En este trabajo, las hipótesis son: a) la nula $\mathrm{H}_{0}$ : no existe correlación entre los resultados obtenidos por las empresas de los subsectores (medidos por ingresos bajos, medios y altos) y el grado de adopción baja, media y alta de las tecnologías de la información y comunicación y b) la alternativa $\mathrm{H}_{1}$ : existe correlación entre los resultados de los subsectores (medidos por ingresos bajos, medios y altos) y el grado de adopción baja, media y alta de las TIC.

En la figura 9 se muestran los resultados de la prueba de hipótesis y del coeficiente de correlación generado por el programa spss; el valor calculado para el coeficiente de correlación tau-b de Kendall fue 0.136 entre las variables analizadas, con un valor $\mathrm{p}$ de 0.037 , y puesto que éste es menor a la significancia estadística para una prueba de dos colas, de 0.05, se rechaza la hipótesis nula. Por lo tanto, se acepta la hipótesis alternativa. Como el valor es positivo, indica que ambas variables se mueven en el mismo sentido, es decir, una mayor adopción de las tecnologías de la información y comunicación se reflejaría en mejores resultados de las empresas de los subsectores, medidos a través de los ingresos. Sin embargo, el coeficiente fue sólo de 0.136 , por lo tanto se concluye que existe una correlación entre el grado de adopción de las TIC y el de incremento de los ingresos de las empresas, pero es muy bajo. 


\section{Figura 9}

Prueba tau-b de Kendall, coeficiente y prueba de hipótesis

\begin{tabular}{|c|c|c|c|c|}
\hline \multicolumn{5}{|c|}{ Correlaciones } \\
\hline & & & Ingreso & Adopción \\
\hline \multirow{6}{*}{$\begin{array}{l}\text { Tau-b de } \\
\text { Kendall }\end{array}$} & \multirow{3}{*}{ Ingreso } & Coeficiente de correlación & 1.000 & $.136^{*}$ \\
\hline & & $\begin{array}{l}\text { Significante para una prueba } \\
\text { de dos colas }\end{array}$ & & .037 \\
\hline & & $\mathrm{N}$ & 191 & 191 \\
\hline & \multirow{3}{*}{ Adopción } & Coeficiante de correlación & $.136^{*}$ & 1.000 \\
\hline & & $\begin{array}{l}\text { Significante para una prueba } \\
\text { de dos colas }\end{array}$ & .037 & \\
\hline & & $\mathrm{N}$ & 191 & 191 \\
\hline
\end{tabular}

* La correlación es significante para una prueba de dos colas a 0.05.

Fuente: elaboración propia, con base en datos obtenidos de la aplicación del cuestionario sobre la adopción de las TIC en PYMEs de Baja California (Ibarra et al. 2013).

Por lo tanto, se puede concluir que la adopción de tecnologías de la información y comunicación influye positivamente en los resultados de las empresas, medidos a través de los ingresos.

\section{Conclusiones}

El análisis arrojó que la adopción de tecnologías entre los subsectores de Baja California es muy baja en las PYMEs; los resultados muestran que no hay interés para invertir en ellas, porque la mayoría de los empresarios las ven más como gasto que como inversión, al no obtener beneficios en el corto plazo.

Esta apreciación constituye un problema, ya que si las empresas no muestran un interés en el aprovechamiento de las TIC, no pueden beneficiarse de las oportunidades que traen consigo. De acuerdo con la evidencia empírica mundial, se sabe que actualmente las TIC son indispensables en el mundo globalizado, debido a los efectos positivos que tienen en muchos aspectos de la vida en general pero, sobre todo, en la productividad y el crecimiento. En las empresas favorece 
la comunicación interna; disminuye los costos de transacción, al fomentar la interacción con clientes y proveedores; reduce los costos de transacción; incrementa la productividad, al estimular la comunicación interna, el uso de técnicas y métodos, y el acceso a mayor información del mercado permite tomar decisiones adecuadas, lo que propicia el ingreso a otros mercados.

Asimismo, se identificaron los subsectores con mayor grado de adopción de tecnologías de la información y comunicación, y se encontró que fue el 5 , correspondiente a derivados del petróleo y del carbón, la industria química del plástico y hule, el 7, de industrias metálicas, y el 8 , de maquinaria y equipo los que han invertido más en ellas.

Las TIC generaron cambios en la comunicación interna, sin embargo no hubo movimientos en cuanto a la creación o desaparición de puestos de trabajo, ni en la calificación de los trabajadores, lo que contrasta con la evidencia empírica. Como las PYMES de Baja California quizá no han puesto interés suficiente en la adopción de las TIC, no se han observado estos cambios o, como demuestra Krueger (1991), existe una correlación entre éstas y la mano de obra calificada, pero no necesariamente una causalidad.

En lo que respecta al entorno global de los subsectores de la manufactura de Baja California, como consecuencia de la adopción de las tecnologías, en general todos resultaron beneficiados, aunque a escala limitada, ya que han logrado una reducción de costos, y también un incremento en sus ingresos. El acceso a otros mercados es visible en los subsectores, pues todos tienen comercio internacional; sin embargo, varía el grado de participación, lo cual no sólo se puede deber, en parte, a la utilización de las TIC sino también a la ubicación geográfica del estado, ya que su cliente principal y competidor externo es Estados Unidos.

También se logró identificar a las TIC más utilizadas para interactuar con los clientes y proveedores. El medio más empleado fue el correo electrónico, seguido por el teléfono y la página de internet.

Asimismo, al correlacionar el grado de adopción de las TIC con los resultados económicos, medidos a través de los ingresos, se encontró que sí existe correlación, pero que es sólo de 0.136 , debido a que en realidad el empleo de éstas ha sido muy limitado en todos los 
subsectores de la manufactura de Baja California, lo que no permite aprovechar los efectos positivos que su implementación genera en la economía. Aunado al hecho de una subutilización de las TIC, como mostraron los resultados, las empresas sólo usan funciones básicas de hardware, software y medios de comunicación con clientes y proveedores, por lo cual es necesaria la capacitación que favorezca el aprovechamiento máximo de ellas.

Las preguntas que surgen son: ¿cuánto tiempo pasará para que los empresarios vean a las TIC como una inversión y no como un gasto?, ¿puede el gobierno facilitar la adopción de las TIC, a través de políticas públicas o programas de fomento?, ¿qué hace falta para utilizar tecnologías más sofisticadas en la interacción con clientes y proveedores?

Investigaciones futuras deberán abordar esta temática en un contexto a escala nacional y multisectorial, ya que la adopción tecnológica no deberá ser exclusiva del sector manufacturero, sino de toda la economía. Es importante destacar que el gobierno, los empresarios y los trabajadores tienen un reto por delante: insertar a México en la globalización tecnológica, como ya se ha hecho en décadas anteriores en el aspecto económico y financiero. Para ello, primero es necesario incrementar el gasto en investigación y desarrollo, que actualmente es de 0.4 por ciento del PIB, cuando debería ser de 1 , además, se requiere elevar la calidad del sector educativo, para que el futuro capital humano del país cuente con las habilidades y destrezas para usar y aprovechar al máximo las TIC; de lo contrario, el desconocimiento, el statu quo de buena parte del sector productivo y la resistencia al cambio impedirán la adopción de las TIC, como parte fundamental del desarrollo económico del país y de sus empresas.

Las universidades deben darle un gran impulso a la adopción tecnológica en el país, a través de sus centros de investigación, sus programas de extensión y vinculación con el sector productivo y también por medio de los estudios de posgrado en esta materia. Con ello se podrá desarrollar capital humano que promueva las TIC dentro de las organizaciones, y elevar así el crecimiento económico de México. 


\section{Bibliografía}

Barrenetxe, Miren y Antonio Cardona. 2013. La brecha digital como fuente de nuevas desigualdades en el mercado de trabajo. Revista del Colegio de Economistas de Madrid 137: 119-128.

Banco Bilbao Vizcaya Argentaria. 2012. Industria automotriz clave en el crecimiento de México. Banco Bilbao Vizcaya Argentaria Research. https://www.bbvaresearch.com/wp-content/uploads/ migrados/120125_PresentacionesMexico_81_tcm346-285045. pdf (10 de febrero de 2013).

Centro de Estudios de las Finanzas Públicas. 2004. Evolución del sector manufacturero de México, 1980-2003. Cámara de Diputados. México. http://www.cefp.gob.mx/intr/edocumentos/pdf/cefp/ cefp0022005.pdf (15 de junio de 2014).

Deloitte. 2010. Índice global de competitividad en manufactura. http://www.deloitte.com.mx/Borderlink/11/january/mx\%28 es-mx\%29IGMD.pdf (11 de enero de 2013).

Federal Reserve Bank of Atlanta. 2013. Próximos a un auge: el sector manufacturero en México construye su futuro Eco South. https:// www.frbatlanta.org/-/.../13q1mexicomanufacturingspanish.pdf (primer cuatrimestre de 2013).

FEM. 2012. The global information technology report: living in a hyperconnected world. http://www3.weforum.org/docs/Global_ IT_Report_2012.pdf (18 de marzo de 2014).

FEM. 2010. The global competitiveness report 2010-2011. http:// www3.weforum.org/docs/WEF_GlobalCompetitivenessReport_2010-11.pdf (8 de agosto de 2012).

FEM. 2009. http://www3.weforum.org/docs/WEF_GlobalCompetitivenessReport_2009-10.pdf 
García, Jacinto. 2002. Consecuencias de las TIC en la economía. NEconomía. Centro de Predicción Económica. http://www.n-economia.com/notas_alerta/pdf/ALERTA_NE_02-2002.

Huerta, Heliana y María F. Chávez. 2003. Tres modelos de política económica en México durante los últimos sesenta años. Revista Análisis Económico XVIII (37): 55-80.

Ibarra, Manuel, Lourdes González y Karla Cervantes. 2013. La adopción de las tecnologías de la información en las PYMEs del sector manufacturero de Baja California. Ponencia presentada en el XVIII Congreso internacional en contaduría, administración e informática, Universidad Nacional Autónoma de México.

INEGI. 2016. Directorio estadístico nacional de unidades económicas. http://www.beta.inegi.org.mx/app/mapa/denue/

INEGI. 2012. Sistema de Cuentas Nacionales. http://www.inegi.org. $\mathrm{mx} / \mathrm{est} / \mathrm{contenidos/proyectos/scn/Informe} \mathrm{preliminar} \mathrm{(5} \mathrm{de} \mathrm{fe-}$ brero de 2013).

INEGI. 2009. Censos económicos. http://www.inegi.org.mx/est/ contenidos/espanol/proyectos/censos/ce2009 (5 de junio de 2014).

INEGI. 2004. Censos económicos. Uso de equipo informático e internet en algunos de los procesos productivos de las empresas. http:/ / www.inegi.org.mx/.../carpetas_tem.aspx?_...Uso_de_equipo_informatico (12 de noviembre de 2013).

Jorgeson, Dale W., Mun S. H. O y Kevin J. Stiroh. 2008. A retrospective look at the U.S.: productivity growth resurgence. Journal of Economic Perspectives 22 (1): 3-24.

Karmarkar, Uday S. y Vandana Mangal. 2004. The business and information technologies (BIT) survey annual report 2003-04. The Business and Information Technologies (BIT) Research Project. 
The Center for Management in the Information Economy (CMIE). The Anderson School of Management at UCLA. BIT Annual Report. www.iese.edu/es/files/BIT_annual\%20report_tcm5-14163.pdf

Krueger, Alan B. 1993. How computers have changed the wage estructure: evidence from microdata, 1984-1989. The Quarterly Journal of Economics 108 (1): 33-60.

Laurencelle, L. 2009. Le tau et le tau b de Kendall pour la correlation de variables ordinals simples ou categorielles. Tutorials in Quantitative Methods for Psychology v (2): 51-58.

Menard, Scott. 2009. Logistic regression: from introductory to advance concepts and applications. Nueva York: SAGE.

OCDE. 2012. ICT skills and employment: new competences and jobs for a greener and smarter economy, OECD digital economy papers. http://www.oecd-ilibrary.org/science-and-technology/ict-skillsand-employment_5k994f3prlr5-en (6 de septiembre de 2014).

OCDE. 2008. Highlights. Information technology outlook 2008. http: //www.oecd.org/internet/ieconomy/41895578.pdf (28 de septiembre de 2014).

OCDE. 2006. ICTs continue to grow strongly, with very rapid growth outside the OECD area. Information technology outlook 2006. http://www.oecd.org/internet/ieconomy/37487604.pdf (16 de noviembre de 2014).

OCDE. 2003. ICT and economic growth. Evidence. Information and communications technologies. http://www.oecdilibrary.org/science-and-technology/ict-and-economicgrowth_9789264101296-en (11 de febrero de 2014).

OCDE. 2000. ICT's, E-commerce and the information economy. http://www.oecd.org/internet/ieconomy/1939833.pdf (5 de septiembre de 2014). 
Oliner, Stephen D. y Daniel E. Sichel. 2000. The resurgence of growth in the late 1990s: is information technology the story? Journal of Economic Perspectives 14 (4): 3-22.

Penn State Eberly College of Science. 2015. Kendall tau-b correlation coefficient. https://onlinecourses.science.psu.edu/stat509/ node/158 (14 de diciembre de 2015).

Secretaría de Desarrollo Económico del Estado de Baja California. 2014. http://www.bajacalifornia.gob.mx/sedeco/cae/cae.html (3 de mayo de 2014).

Solow, Robert. 1956. A contribution to the theory of economic growth. The Quarterly Journal of Economics 70 (1): 65-94.

Statistical Research. 2012. Kendall's tau. http://statistical-research. com/wp-content/uploads/2012/09/kendall-tau1.pdf (13 de diciembre de 2015).

Stiroh, Kevin J. 2002. Information technology and the U. S. productivity revival: what do the industry data say? American Economic Review 92 (5): 1559-1576.

Tello, Edgar. 2008. Las tecnologías de la información y comunicación (TIC) y la brecha digital: su impacto en la sociedad de México. Revista de Universidad y Sociedad del Conocimiento Rusc. www.uoc.edu/ rusc/4/2/dt/esp/tello.html

Thompson, Herbert G. y Christopher Garbacz. 2007. Productivity impacts of mobile versus fixed broadband use. http://businessinnovation.berkeley.edu/Mobile_Impact/Thompson_and_Garbacz. pdf (5 de mayo de 2014).

Valderrama Santibáñez, Ana Lilia y Omar Neme Castillo. 2011. Efectos de las tecnologías de la información y comunicación (TIC) en las exportaciones manufactureras en México. Revista Economía unAM 8 (24): 99-124. 
Wieck, Reinhard y Miguel Vidal. 2010. Investment in telecommunications infrastructure, growth, and employment. Ponencia presentada en la 21st European regional ITS conference, Copenhague.

Zhen-Wei, Qiang Christine y Alexander Pitt. 2003. Contribution of information technologies to growth working paper 24. The World Bank. https://openknowledge.worldbank.org/handle/10986/15 059 
